02

\title{
Поглощение мощного света свободными электронами в кристаллах: внутризонные электрон-фононные осцилляции Раби
}

\author{
(C) Е.Ю. Перлин, А.В. Иванов, А.А. Попов \\ Университет ИТМО, \\ 199034 Санкт-Петербург, Россия \\ e-mail eyperlin@itmo.ru
}

Поступила в редакцию 06.08.2020 г.

В окончательной редакции 06.08.2020 г.

Принята к публикации 25.08.2020 г.

В рамках модифицированного резонансного приближения рассчитано поглощение свободными электронами мощного лазерного излучения видимого либо ближнего ИК диапазона. Получены вероятности $W_{\text {ехс }}$ внутризонных переходов с изменением энергии электрона на $\hbar\left(\omega \pm \omega_{l}\right)$, где $\omega-$ частота света, $\omega_{l}-$ частота продольных оптических фононов, участвующих в процессе. Показано, что могут иметь место специфические электрон-фононные осцилляции Раби с частотой $\Omega_{\mathrm{R}}$, а поглощение света идет только до времени достижения первого максимума $\tau_{1}$ на зависимости $W_{\text {exc }}(t)$, где $t$ - время, прошедшее с начала действия лазерного импульса. Показано, что в случае предпробойных интенсивностей света процессы высоких порядков по полю световой волны существенным образом влияют на $W_{\mathrm{exc}}, \tau_{1}$ и $\Omega_{\mathrm{R}}$.

Ключевые слова: внутризонное поглощение света, оптический эффект Штарка, осцилляции Раби, гамильтониан Фрёлиха, предпробойные интенсивности света.

DOI: $10.21883 /$ OS.2020.12.50321.212-20

\section{1. Введение}

Исследование физических механизмов предпробойного возбуждения и пробоя прозрачных полупроводников и диэлектриков мощным лазерным излучением остается предметом многочисленных исследований в течение ряда десятилетий (см., например, [1-12]). Существенную роль в запуске процессов, ведущих к пробою материала, играет поглощение мощного лазерного излучения свободными носителями заряда, появляющимися в зоне проводимости и валентной зоне за счет многофотонных межзонных переходов либо ударной ионизации кристаллической решетки. При поглощении света свободными электронами они приобретают кинетическую энергию, достаточную для ударной ионизации либо для комбинированной многофотонно-ударной ионизации $[13,14]$. Эти процессы могут вызвать лавинообразное нарастание концентрации свободных носителей заряда.

Поглощению света свободными электронами посвящено большое число работ (см., например, [15-23]). Как правило, при теоретическом рассмотрении непрямых внутризонных переходов используется второй порядок теории возмущений - один порядок по взаимодействию электрона с полем электромагнитной волны, а второй по взаимодействию электрона с фононами либо потенциалом заряженных примесей ${ }^{1}$. Прямые внутризонные оптические переходы невозможны, так как для них не могут одновременно выполняться законы сохранения энергии и импульса.

\footnotetext{
${ }^{1}$ В работе [15], а затем и в учебниках [16] и [17] приведены неточные формулы для вероятности перехода во втором порядке теории возмущений.
}

Нелинейное поглощение света и динамика электронов в присутствии мощного лазерного излучения рассматривались в работах [24-33]. Существенным обстоятельством, которое необходимо учитывать при описании одно- и многофотонного внутризонного поглощения сверхкоротких импульсов лазерного излучения предпробойной интенсивности, является неадекватность представления о вероятности перехода за единицу времени [30]. При этом возникает вопрос о возможности осцилляций Раби и проявлений резонансного оптического эффекта Штарка (ОЭШ) на непрямых внутризонных переходах.

ОЭШ (или эффект Отлера-Таунса [31]) в твердых телах исследовался в многочисленных работах (см., например, [32-60]). В работах [32-34] было показано, что в случае, когда в некоторой точке зоны Бриллюэна энергия кванта света $\hbar \omega$ совпадает с энергетическим зазором между зонами, в зависимости от параметров электронной зонной структуры происходит расщепление или разрыв зон, причем величина щели пропорциональна напряженности поля электромагнитной волны. В работах [35,37] предсказывалось появление системы щелей в энергетическом зонном спектре кристалла под действием лазерного излучения очень высокой интенсивности, причем в [35], а позже в [52] электронная зонная структура с самого начала была рассчитана с учетом сильного электромагнитного поля. Влияние ОЭШ при резонансе на переходе между двумя зонами проводимости на возбуждение нанокристаллов $\mathrm{AgBr}$ исследовано в работе [51]. Значительная часть работ по ОЭШ посвящена экситонным системам. В работах $[36,38,48]$ 
рассматривался случай, когда частота света попадает в резонанс между состоянием дискретного (экситонного) спектра и одним из состояний непрерывного зонного спектра. В этом случае проявляется резонанс Фано [49] в электрон-фотонной системе. В работе [39] исследовался ОЭШ под действием длинноволнового излучения $\mathrm{CO}_{2}$-лазера при резонансе на переходах между экситонными состояниями $1 s$ и $2 p$ в $\mathrm{Cu}_{2} \mathrm{O}$. В работе [40] по ОЭШ в квантовых точках и большинстве других работ по ОЭШ (см., например, [40-42,50,55$60]$ ) использовалось лазерное излучение с частотой, несколько меньшей ширины запрещенной зоны. В работах $[43,44]$ исследовались сдвиги экситонных уровней с учетом многочастичных эффектов за счет заполнения зон при двухфотонных межзонных переходах. В работах $[57,59]$ изучался ОЭШ в экситонно-биэкситонных системах. Поляризационно-зависимый ОЭШ стал предметом изучения в [57-59]. В работах $[45,46,52,53]$ рассматривался ОЭШ в условиях двойного однофотоннооднофотонного [46], двухфотонно-однофотонного [45] и многофотонно-двухфотонного резонанса на смежных межзонных переходах. Было показано, что в перестроенном благодаря взаимодействию с сильным светом электронном зонном спектре появляются дополнительные сингулярности Ван-Хова. Положение этих сингулярностей в зоне Бриллюэна зависит от интенсивности света $j_{\omega}$. В результате на графиках зависимости скорости многофотонной генерации неравновесных электроннодырочных пар $W_{\mathrm{vc}}$ от $j_{\omega}$ появляются области, где малое (на единицы\%) изменение $j_{\omega}$ приводит к изменению $W_{\text {vс }}$ на несколько порядков величины, что, в свою очередь, может привести к запуску процесса оптического пробоя. В работе [55] предлагается использовать ОЭШ для управления переносом энергии в двухуровневых диссипативных системах в новом механизме лазерного охлаждения.

При высоких интенсивностях лазерного излучения осцилляции Раби и ОЭШ могут иметь место не только в случае однофотонного резонанса, но и в случае многофотонных (в том числе двухфотонных) резонансов $[60,61,52]$. В настоящей работе исследуются осцилляции Раби при двухквантовых (фотон+фонон) осцилляциях Раби при внутризонных переходах с участием продольных оптических (LO) фононов под действием мощного лазерного излучения видимого или ближнего ИК диапазона.

\section{2. Гамильтониан электрон-фононной системы в поле электромагнитной волны}

Представим $\hat{H}$ электрон-фононной системы в поле электромагнитного излучения в виде

$$
\hat{H}=\hat{H}_{0}+\hat{H}^{\prime}, \quad \hat{H}_{0}=\hat{H}_{0}^{(\text {el })}+\hat{H}_{0}^{(\text {phon })},
$$

$$
\hat{H}^{\prime}=\hat{H}_{\mathrm{e} \text {-phot }}^{\prime}+\hat{H}_{\text {phon }}^{\prime},
$$

где $\hat{H}_{0}^{(\text {el) }}$ и $\hat{H}_{0}^{\text {(phon) }}-$ гамильтонианы невзаимодействующих электронной и фононной подсистем:

$$
\begin{gathered}
\hat{H}_{0}^{(\mathrm{el})} \psi_{\mathbf{k}}=E_{c}(\mathbf{k}) \psi_{\mathbf{k}}, \\
\hat{H}_{0}^{(\text {phon })}=\hbar \omega_{l} \sum_{\mathbf{q}} b_{\mathbf{q}}^{+} b_{\mathbf{q}} .
\end{gathered}
$$

В формулах (3), (4) $\psi_{\mathbf{k}}$ и $E_{c}(\mathbf{k})-$ блоховская функция и блоховская энергия электронов в зоне проводимости, $\omega_{l}$ - предельная частота LO-фононов, дисперсию которых мы не учитываем, $b_{\mathbf{q}}^{+}$и $b_{\mathbf{q}}-$ операторы рождения и уничтожения LO-фононов с волновым вектором q. Оператор взаимодействия электрона с полем электромагнитной волны имеет вид

$$
\begin{gathered}
\hat{H}_{\mathrm{e}-\mathrm{phot}}^{t}=\hat{H}_{\mathrm{e}-\text { phot }}^{(1)}+\hat{H}_{\mathrm{e} \text {-phot }}^{(2)}, \\
\hat{H}_{\mathrm{e} \text {-phot }}^{(1)}=-\frac{e}{2 m c}(\mathbf{A} \cdot \hat{\mathbf{q}}+\hat{\mathbf{q}} \cdot \mathbf{q}), \\
\hat{H}_{\mathrm{e}-\text { phot }}^{(2)}=\frac{1}{2}\left(\frac{e}{m c}\right)^{2} \mathbf{A}^{2},
\end{gathered}
$$

где $e$ и $m-$ заряд и масса свободного электрона, $c-$ скорость света, $\hat{\mathbf{q}}-$ оператор импульса, действующий в электронной подсистеме, $\mathbf{A}$ - вектор-потенциал электромагнитного поля, который мы представим в виде

$$
\mathbf{A}(\mathbf{r})=\operatorname{Re}\left[A_{0} \mathbf{e}_{\mathbf{k}} e^{-i(\omega t-\mathbf{k} \cdot \mathbf{r})}\right] .
$$

В формуле (5) $\mathbf{e}_{\mathbf{k}}-$ единичный вектор поляризации света, $\mathbf{k}-$ волновой вектор, $\omega-$ частота света. Амплитуду $A_{0}$ вектора-потенциала можно выразить через интенсивность излучения $j_{\omega}$ с помощью соотношения

$$
A_{0}^{2}=\frac{8 \pi c}{\sqrt{\varepsilon_{\infty}} \omega^{2}} j_{\omega}
$$

где $\varepsilon_{\infty}-$ высокочастотная диэлектрическая проницаемость.

Взаимодействие электронной подсистемы с оптическими фононами описывается гамильтонианом Фрёлиxa [62]

$$
H_{\mathrm{e}-\mathrm{phon}}^{\prime}=\frac{\vartheta}{\sqrt{\Omega}} \sum_{\mathbf{q}} q^{-1}\left(b_{\mathbf{q}}-b_{\mathbf{q}}^{+}\right) e^{i \mathbf{q} \cdot \mathbf{r}},
$$

где $\Omega$ - нормировочный объем,

$$
\vartheta=-i e \sqrt{2 \pi \hbar \omega_{l} / \varepsilon^{*}}, \quad 1 / \varepsilon^{*}=1 / \varepsilon_{0}-1 / \varepsilon_{\infty},
$$

$\varepsilon_{0}-$ статическая диэлектрическая проницаемость.

Квадратичный по полю член в правой части (4) не приводит к однофотонным внутризонным переходам. В пренебрежении малым волновым вектором фотона роль этого члена сведется к изменению энергии электрона на величину, пропорциональную интенсивности 
света $j_{\omega}$. Мы приближенно учтем это обстоятельство введением эффективной электронной температуры

$$
T_{\mathrm{eff}}=T_{0}+\Delta T, \quad \Delta T==\frac{8 \pi j_{\omega}}{3 c k_{\mathrm{B}} m_{c} \omega^{2}},
$$

где $T_{0}$ - температура решетки, которая не успевает измениться за короткое время действия мощного лазерного импульса, $k_{\mathrm{B}}$ - постоянная Больцмана. Отметим, что в результате стандартной процедуры исключения межзонной части взаимодействия электрона в зоне $c$ c полем световой волны в квадратичном члене в правой части (4) и в правой части (9) вместо массы свободного электрона $m$ появляется эффективная масса $m_{c}$.

\section{3. Расчет вероятности возбуждения электронной системы}

Вероятность $W_{\text {ехс }}$ того, что в момент времени $t$ электрон перейдет из группы состояний с волновыми векторами $\mathbf{k}$ и энергиями $E_{c}(\mathbf{k})$ вблизи дна зоны проводимости $c$ в возбужденные состояния с волновыми векторами $\mathbf{k} \pm \mathbf{q}$ и энергией $E_{c}(\mathbf{k} \pm \mathbf{q})=E_{c}(\mathbf{k})+\hbar \omega \pm \hbar \omega_{l}$, дается выражением

$$
W_{\mathrm{exc}}=\left\langle\sum_{\mathbf{q}}\left|\left\langle\mathbf{k} \pm \mathbf{q}, n_{\mathbf{q}} \mp 1\left|e^{-i \hat{H} t / \hbar}\right| \mathbf{k}, n_{\mathbf{q}}\right\rangle\right|\right\rangle_{\mathrm{el}, \text { phon }},
$$

где $n_{\mathbf{q}}$ - числа заполнения фононов, $\langle\ldots\rangle$ el,phon усреднение по состояниям электронной и колебательной подсистем кристалла.

Воспользуемся дважды известным соотношением для распутывания экспоненциальных операторов

$$
\begin{gathered}
e^{-i\left(\hat{H}_{0}+\hat{H}_{\mathrm{e} \text {-phon }}^{(1)}+H_{\mathrm{e} \text {-phon }}^{\prime}\right) t / \hbar}=e^{-i\left(\hat{H}_{0}+\hat{H}_{\mathrm{e} \text {-phon }}^{(1)}\right) t / \hbar} \hat{S}, \\
\hat{S}=T \exp \left\{-(i / \hbar)^{n} \int_{0}^{t} e^{i\left(\hat{H}_{0}+\hat{H}_{\mathrm{e} \text {-phot }}^{(1)}\right) t_{1} / \hbar}\right. \\
\left.\times \hat{H}_{\mathrm{e} \text {-phon }}^{\prime} e^{-i\left(\hat{H}_{0}+\hat{H}_{\mathrm{e} \text {-phot }}^{(1)}\right)_{1} / \hbar} d t_{1}\right\} \\
e^{-i\left(\hat{H}_{0}+\hat{H}_{\mathrm{e} \text {-phot }}^{(1)}\right) t / \hbar}=e^{-i \hat{H}_{0} t / \hbar} \hat{g}(t), \\
\hat{g}(t)=T \exp \left\{-(i / \hbar)^{n} \int_{0}^{t} e^{i \hat{H}_{0} t_{1} / \hbar}\right. \\
\left.\times \hat{H}_{\mathrm{e}-\mathrm{phot}}^{(1)} e^{-i \hat{H}_{0} t_{1} / \hbar} d t_{1}\right\}
\end{gathered}
$$

где $T$ обозначает хронологическое упорядочение операторов.

В пренебрежении импульсом фотона $\mathbf{k}$ у оператора $\hat{g}(t)$ отличны от нуля лишь диагональные матричные элементы, которые легко вычисляются. Пусть вектор поляризации света $e_{\mathrm{k}}$ направлен вдоль оси $x$. С учетом соотношения

$$
\mathbf{P}_{c \mathbf{k}, c \mathbf{k}}=\frac{m}{m_{c}} \hbar \mathbf{k}
$$

где $m_{c}$ - эффективная масса электрона, имеем для диагональных матричных элементов $\hat{g}(t)$ :

$$
g_{k_{x} k_{x}}(t)=\exp \left[i \xi\left(k_{x}\right) \sin \omega t\right]=\sum_{n=-\infty}^{\infty} J_{n}\left[\xi\left(k_{x}\right)\right] e^{i n \omega},
$$

где $J_{n}(\zeta)$ - функции Бесселя (см., например, [63]),

$$
\xi\left(k_{x}\right)=\frac{2 e k_{x}}{m_{c} \omega^{2}} \sqrt{\frac{2 \pi j_{\omega}}{c \sqrt{\varepsilon_{\infty}}}} .
$$

Для упрощения задачи предположим, что зона проводимости достаточно узкая, так что двухфотонные внутризонные переходы невозможны. Предположим также, что частота света $\omega$ намного превышает частоту оптических колебаний решетки $\omega_{l}$, соответственно длина $q$ волнового вектора намного превосходит длину $k_{1} x$ компоненты волнового вектора электрона в начальном состоянии:

$$
\omega \gg \omega_{l}, \quad q \gg k_{1}, \quad \hbar \omega \gg k_{\mathrm{B}} T_{\mathrm{eff}}
$$

При выполнении условий (16) приближенно получим

$$
k_{1} \approx \frac{1}{\hbar} \sqrt{m_{c} k_{\mathrm{B}} T_{\mathrm{eff}}}, \quad k_{2} \approx \sqrt{\frac{m_{c} \omega}{3 \hbar}},
$$

где $k_{2}$ - длина вектора $x$-компоненты волнового вектора электрона в возбужденном состоянии (далее для сокращения записи будем обозначать основное и возбужденное состояния электрона в зоне проводимости индексами $i$ и $f$ ).

Согласно (11), (12), получим для оператора эволюции следующее выражение:

$$
\begin{aligned}
\hat{S}= & T \exp \left\{-(i / \hbar)^{n} \int_{0}^{t} \hat{g}^{+}\left(t_{1}\right) e^{i \hat{H}_{0}^{(\mathrm{el})} t_{1} / \hbar}\right. \\
& \left.\times H_{\mathrm{e}-\text { phon }}^{\prime}\left(t_{1}\right) e^{-i \hat{H}_{0}^{(\mathrm{el})} t_{1} / \hbar} \hat{g}\left(t_{1}\right) d t_{1}\right\}, \\
H_{\mathrm{e}-\text { phon }}^{\prime}\left(t_{1}\right) & =e^{i \hat{H}_{0}^{(\text {phon })} t_{1} / \hbar} H_{\mathrm{e}-\text { phon }}^{\prime}\left(t_{1}\right) e^{-i \hat{H}_{0}^{(\text {phon })} t_{1} / \hbar} .
\end{aligned}
$$

Введем обозначения

$$
\delta_{1,2}(\mathbf{k}, \mathbf{q})=\frac{1}{\hbar}\left[E_{c}(\mathbf{k} \pm \mathbf{q})-E_{c}(\mathbf{k})\right]-\omega \pm \omega_{l}
$$

для процессов с поглощением (1) либо испусканием (2) LO-фонона. Для параболического закона дисперсии $E_{c}(\mathbf{k})=\hbar^{2} k^{2} /\left(2 m_{c}\right)$ при выполнении условий $(16)$

$$
\delta_{1,2}(\mathbf{k}, \mathbf{q}) \approx \delta_{1.2}(q)=\frac{\hbar q^{2}}{2 m_{c}}-\omega \pm \omega_{l} .
$$

Матричный элемент $S_{f i}$ оператора $\hat{S}$ (18) будем вычислять в резонансном приближении (приближении вращающегося поля). Это означает, что при разложении в ряд $T$-экспоненты в правой части (18) мы будем 
сохранять в каждом порядке члены, где в показателе экспоненты фигурируют величины $i \delta_{1,2}(\mathbf{q}) t$, чередующиеся с $-i \delta_{12}(\mathbf{q}) t$. В резонансном приближении в каждом из интегралов по $d t_{n}$ появляется, согласно (14), множитель

$$
v=\sum_{n=-\infty}^{\infty} J_{n}\left(\xi_{1}\right) J_{n+1}\left(\xi_{2}\right) \text {. }
$$

Воспользовавшись теоремой сложения Неймана для функций Бесселя [63], преобразуем (21) к виду

$$
v=J_{1}\left(\xi_{2}-\xi_{1}\right),
$$

причем в случае высоких интенсивностей света $j_{\omega} \sim 10^{11}-10^{13} \mathrm{~W} / \mathrm{cm}^{2}$ и частот лазерного излучения $\omega \sim 0.3 \cdot 10^{15}-3 \cdot 10^{15} \mathrm{~s}^{-1}$ аргументы функций Бесселя $\xi_{1}=\xi\left(k_{1}\right)$ и $\xi_{2}=\zeta\left(k_{2}\right)$ могут значительно превышать единицу. Множитель $v$ описывает влияние процессов высших порядков на частоту внутризонных электронфононных осцилляций Раби.

Нетрудно убедиться в том, что основной вклад в суммирование по волновым векторам фонона q в правой части (10) дает достаточно узкая область вблизи значений $q$, при которых $\delta_{1,2}(q)=0$. При выполнении условий (16) ширина этой области $\Delta q \sim 2 k_{1}$. В пределах этой области неэкспоненциальная часть подынтегрального выражения в каждой из сумм по q изменяется слабо.

В разложении $S_{f i}$ в ряд по степеням $H_{\mathrm{e}-\mathrm{phon}}^{\prime}$ coхраняются лишь члены нечетного $(2 n+1)$-го порядка. Заменим приближенно усреднение каждого члена разложения $S_{f i}$ на произведение попарно усредненных операторов в интегралах по $d t_{2 n}$ и $d t_{2 n+1}$ :

$$
\begin{aligned}
& \Xi^{2}=\left\langle\sum_{\mathbf{q}}\left|\left\langle\mathbf{k} \pm \mathbf{q}, n_{\mathbf{q}} \mp 1\left|H_{\text {e-phon }}^{\prime}\right| \mathbf{k}, n_{\mathbf{q}}\right\rangle\right|^{2}\right\rangle_{\text {phon }} \\
& =\frac{1}{(2 \pi)^{3}}|\vartheta|^{2} \int d \mathbf{q} \frac{1}{q^{2}}\left\{\begin{array}{l}
n_{\mathbf{q}} \\
n_{\mathbf{q}}+1
\end{array}\right. \\
& \approx \frac{4 \pi}{(2 \pi)^{3}}|\vartheta| \int_{q_{0}}^{q_{0}+\Delta q} d q\left\{\begin{array} { l } 
{ n _ { 1 } } \\
{ n _ { l } + 1 }
\end{array} \approx \frac { | \vartheta | ^ { 2 } } { \pi ^ { 2 } } k _ { 1 } \left\{\begin{array}{l}
n_{l} \\
n_{l}+1
\end{array},\right.\right.
\end{aligned}
$$

где $n_{l}=\left\{\exp \left[\hbar \omega_{l} /\left(k_{\mathrm{B}} T\right)\right]-1\right\}^{-1}$.

В принятых приближениях матричный элемент $S_{f i}^{s}$ (верхние индексы $s=1$ и $s=2$ соответствуют процессам с поглощением и испусканием LO-фонона) является решением уравнения

$$
\ddot{S}_{f i}^{(s)}-i \delta_{s} \dot{S}_{f i}^{(s)}+\left|P_{s}\left(k_{1}\right)\right|^{2} S_{f i}^{(s)}=0
$$

с начальными условиями

$$
\left.S_{f i}^{(s)}\right|_{t=0}=1,\left.\quad \dot{S}_{f i}^{(s)}\right|_{t=0}=-i e^{i \delta_{s}(q) t} \tilde{P}_{s \mathbf{q}},
$$

где

$$
P_{s}=\frac{v \vartheta}{\hbar} \sqrt{\frac{k_{1} \eta_{s}}{\pi}}, \quad \tilde{P}_{s \mathbf{q}}=\frac{v \vartheta}{\hbar} \sqrt{\frac{\eta_{s}}{\Omega}} \sum_{\mathbf{q}} \frac{1}{q},
$$

$$
\begin{aligned}
\eta_{1}=n_{l}, \eta_{2} & =n_{l}+1 \text {. Имеем } \\
S_{f i}^{(s)}(\mathbf{q}, t) & =-i \exp \left[i \frac{\delta_{s}(\mathbf{q})}{2} t\right] \frac{\tilde{P}_{s \mathbf{q}}}{\sqrt{\left[\delta_{s}(\mathbf{q}) / 2\right]^{2}+\left|P_{s}\right|^{2}}} \\
& \times \sin \sqrt{\left[\delta_{s}(\mathbf{q}) / 2\right]^{2}+\left|P_{s}\right|^{2}} t .
\end{aligned}
$$

Подставляя (27) в (10), получим после некоторых вычислений

$$
\begin{aligned}
W_{\text {exc }}(t) & =\frac{1}{2} \int_{0}^{\infty} f(k) k d k \int_{0}^{\infty} \sum_{s=1}^{2} \frac{\left|P_{s}(k)\right|^{2}}{\left|P_{s}(k)\right|^{2}+\left[\frac{1}{2} \delta_{s}(q)\right]^{2}} \\
& \times \sin ^{2}\left\{\sqrt{\left|P_{s}(k)\right|^{2}+\left[\frac{1}{2} \delta_{s}(q)\right]^{2}} t\right\} d q,
\end{aligned}
$$

где

$$
f(k)=\sqrt{\frac{2}{\pi}}\left(\frac{\hbar^{2}}{m_{c} k_{\mathrm{B}} T_{\mathrm{eff}}}\right)^{\frac{3}{2}} \exp \left[-\frac{\hbar^{2} k^{2}}{2 m_{c} k_{\mathrm{B}} T_{\mathrm{eff}}}\right] .
$$

\section{4. Результаты расчетов}

В расчетах используем следующие значения параметров, типичные для кристаллов типа $\mathrm{A}_{2} \mathrm{~B}_{6}$, например, $\mathrm{ZnS}: m_{c}=0.35 m, \omega_{l}=5 \cdot 10^{13} \mathrm{~s}^{-1}, \varepsilon_{0}=8.5, \varepsilon_{\infty}=5.2$. Отметим, что изменение этих параметров в разумных пределах не приводит к изменению качественной картины рассматриваемых процессов.

На рис. $1, a-d$ представлены зависимости вероятности перехода в возбужденные состояния зоны проводимости $W_{\text {exc }}$ от времени, рассчитанные с помощью (28), (29), (26), (22). Мы видим, что после достижения первого пика $W_{\text {exc }}$ происходят осцилляции вокруг некоторого среднего значения.

Обозначим через $W_{\max }$ максимальное значение $W_{\text {exc }}$, которое обычно достигается в первом или втором пике на зависимостях $W_{\text {exc }}(t)$. На рис. 2 приведены зависимости $W_{\max }$ от интенсивности света $j_{\omega}$ при фиксированных значениях частоты света $\omega$.

На рис. 3 показана зависимость времени достижения первого максимума $\tau_{1}$ на зависимости $W_{\max }(t)$ от интенсивности $j_{\omega}$.

Определим также частоту Раби для рассматриваемого процесса как $\Omega_{\mathrm{R}}=2 \pi /\left(\tau_{2}-\tau_{1}\right)$, где $\tau_{2}-$ время, за которое достигается второй максимум на кривой $W_{\mathrm{exc}}(t)$. На рис. 4 даются зависимости $\Omega_{\mathrm{R}}\left(j_{\omega}\right)$.

На рис. 5 приводится зависимость $W_{\max }$ от частоты света $\omega$ для $j_{\omega}=100 \mathrm{GW} / \mathrm{cm}^{2}$.

Сложный характер зависимостей, представленных на рис. 2-5, обусловлен в основном множителем $v(22)$, описывающим вклад процессов высших порядков по полю в эффективный матричный элемент перехода (26).

Определенный интерес представляет случай моноэнергетического распределения электронов в начальном 

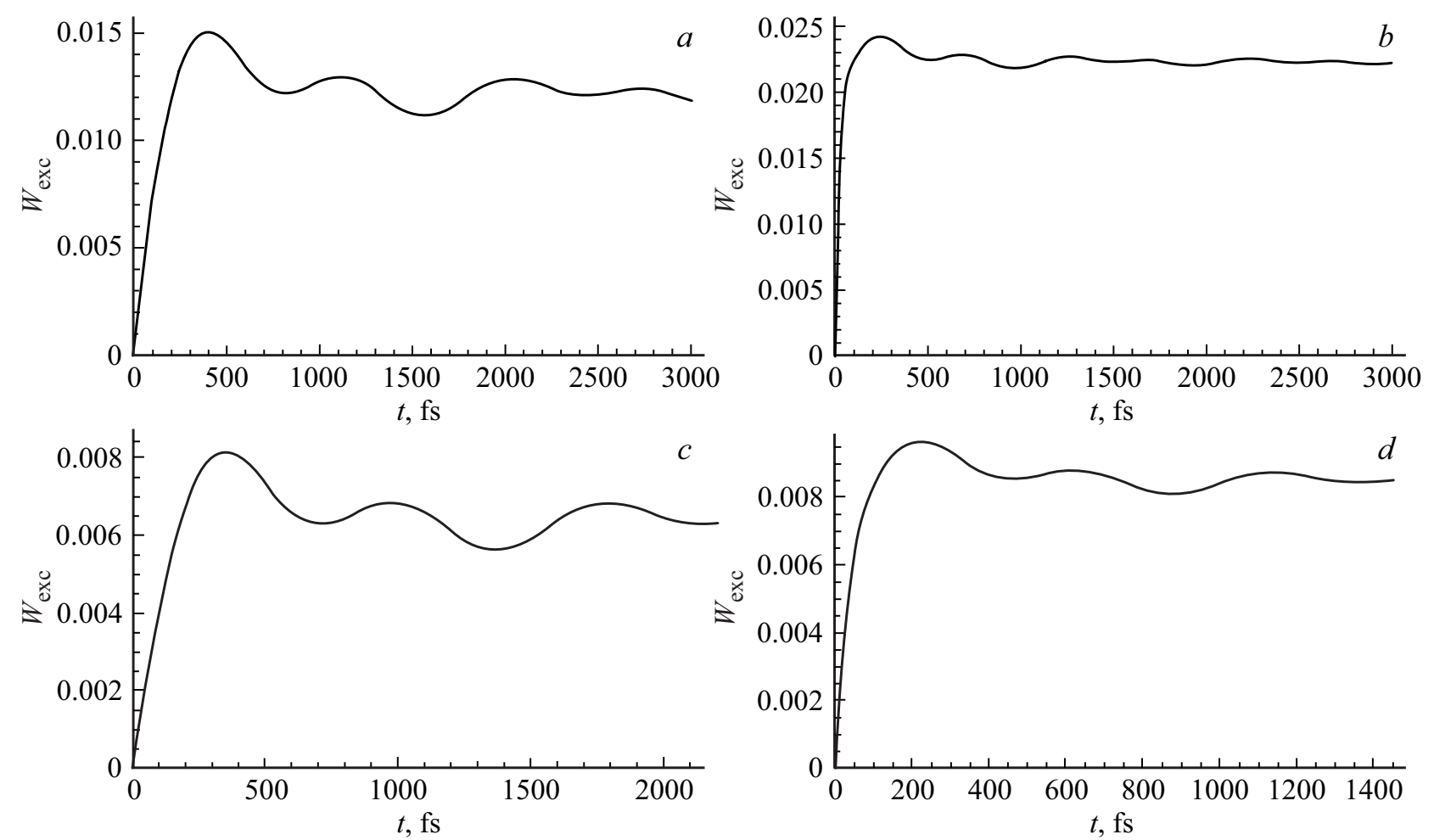

Рис. 1. Зависимости вероятности $W_{\text {ехс }}$ от времени с начала действия лазерного излучения: $(a) j \omega=30 \mathrm{GW} / \mathrm{cm}^{2}, \omega=0.7 \cdot 10^{15} \mathrm{~s}^{-1}$; (b) $j_{\omega}=80 \mathrm{GW} / \mathrm{cm}^{2}, \omega=0.7 \cdot 10^{15} \mathrm{~s}^{-1} ;$ (c) $j_{\omega}=400 \mathrm{GW} / \mathrm{cm}^{2}, \omega=1.78 \cdot 10^{15} \mathrm{~s}^{-1} ;$ (d) $j_{\omega}=1000 \mathrm{GW} / \mathrm{cm}^{2}, \omega=1.78 \cdot 10^{15} \mathrm{~s}^{-1}$.
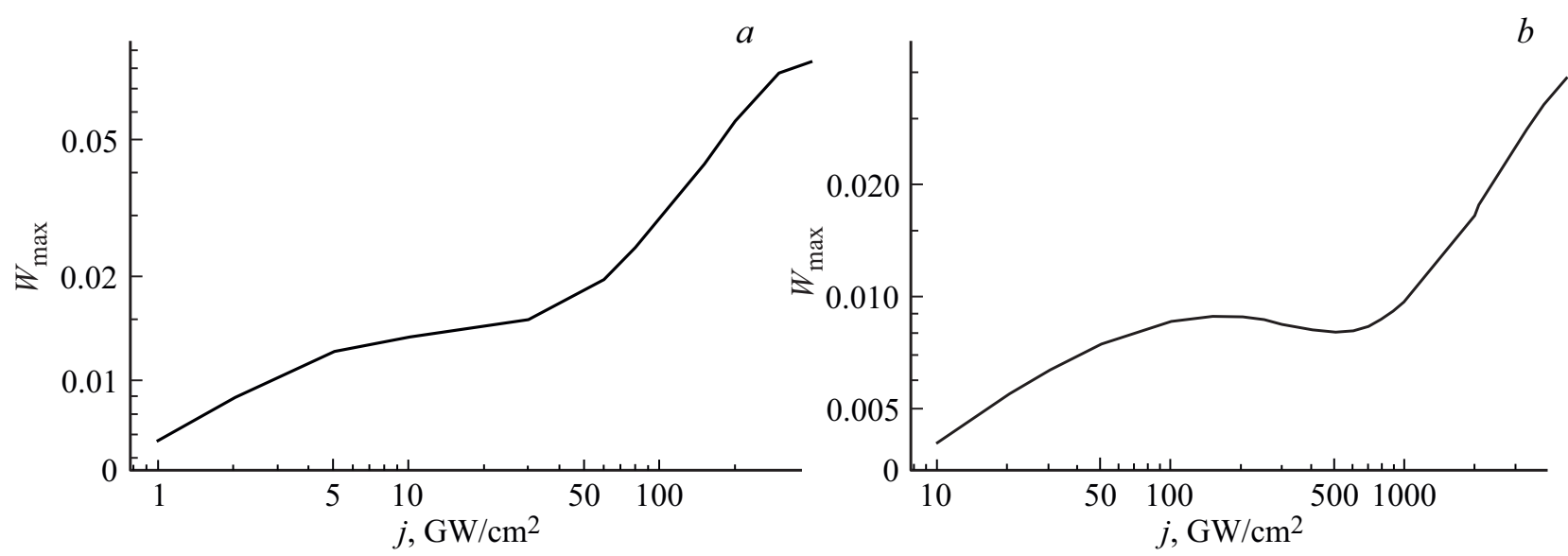

Рис. 2. Зависимости $W_{\max }$ от интенсивности света $j_{\omega}:(a) \omega=0.7 \cdot 10^{15} \mathrm{~s}^{-1} ;(b) \omega=1.78 \cdot 10^{15} \mathrm{~s}^{-1}$.

состоянии, которое может возникнуть в результате прямого оптического межзонного перехода под действием дополнительного света с частотой, большей $\omega$, и сохраняться в течение времени $t<\tau_{\mathrm{r}}$, где $\tau_{\mathrm{r}}-$ время релаксации импульса электрона $\left(\tau_{\mathrm{r}} \sim 10^{-12}-10^{-13} \mathrm{~s}\right)$. На рис. 6, a для этого случая показана типичная зависимость $W_{\max }$ от интенсивности $j_{\omega}$ при фиксированной частоте $\omega=0.7 \cdot 10^{15} \mathrm{~s}^{-1}$, на рис. $6, b-$ при $\omega=1.78 \cdot 10^{15} \mathrm{~s}^{-1}$, а на рис. $6, c-$ зависимость $W_{\max }$ от $\omega$ при фиксированном значении $j_{\omega}=100 \mathrm{GW} / \mathrm{cm}^{2}$. В расчетах использованы значения параметров, указан- ные в начале данного раздела. Кроме того, принимаем, что начальная кинетическая энергия $\delta E=0.1 \mathrm{eV}$; $k_{1}=\hbar^{-1} \sqrt{m_{c}\left(\frac{2}{3} \delta E+\Delta T\right)}$.

Видно, что на кривых $W_{\max }\left(j_{\omega}\right)$ и $W_{\max }(\omega)$ имеются узкие провалы вплоть до нулевых значений $W_{\max }$. Эти провалы соответствуют нулевым (или очень малым) значениям множителя $v$ (см. формулы (21), (22), (26)). Этот множитель, как уже отмечалось, описывает вклад процессов высших порядков по полю световой волны в вероятность возбуждения зонных электронов. Очевидно, что в актуальном для рассматриваемых процессов диапа- 

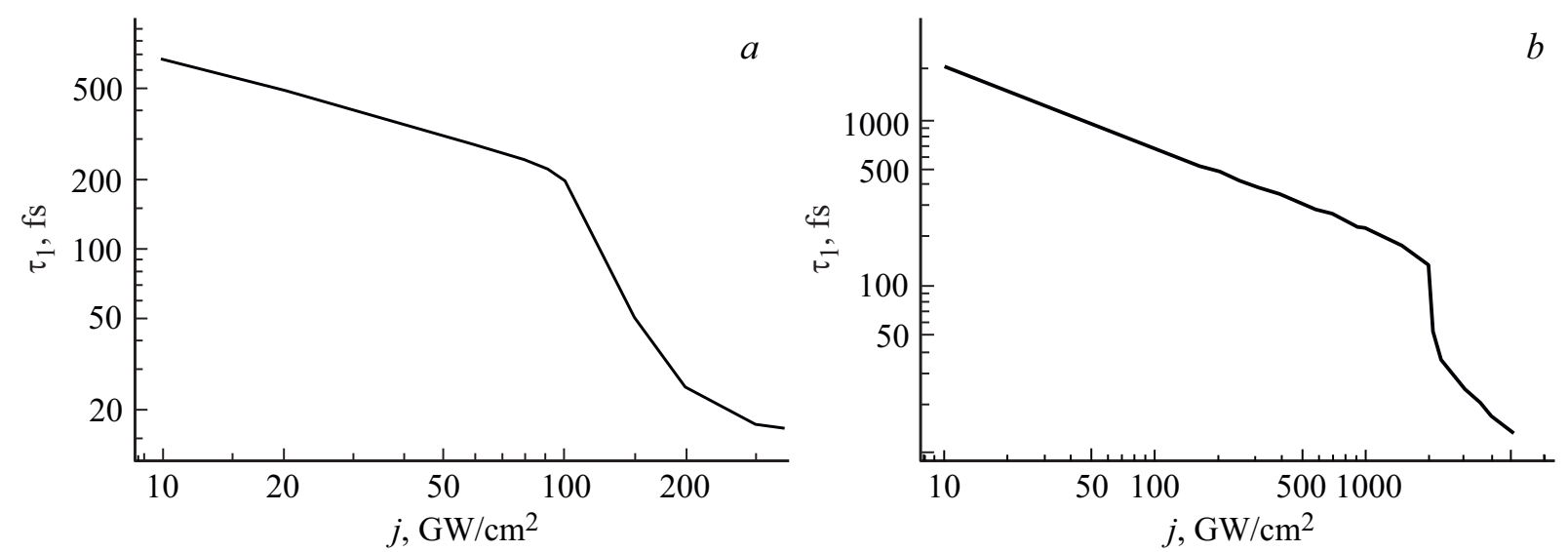

Рис. 3. Зависимости времени достижения 1-го максимума на кривой $W_{\text {ехс }}(t)$ от интенсивности $j_{\omega}:(\mathrm{a}) \omega=0.7 \cdot 1015 \mathrm{~s}^{-1}$; (b) $\omega=1.78 \cdot 1015 \mathrm{~s}^{-1}$.
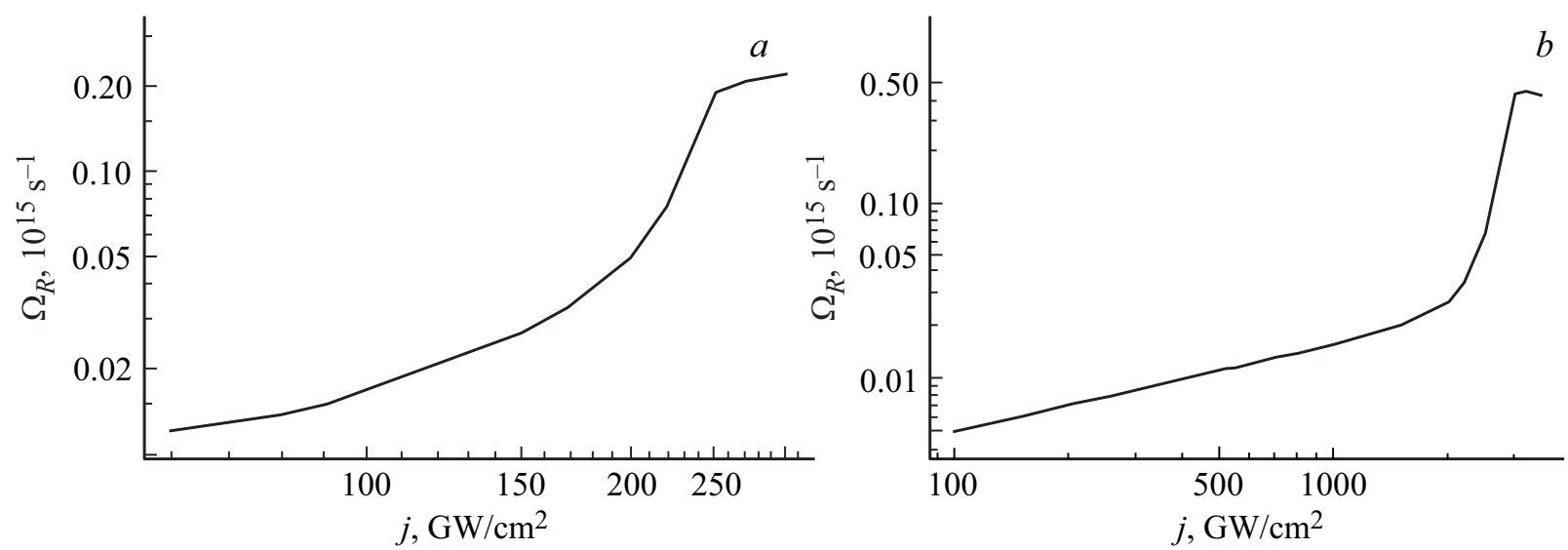

Рис. 4. Зависимости частоты Раби $\Omega_{\mathrm{R}}$ от интенсивности $j_{\omega}:(a) \omega=0.4 \cdot 10^{15} \mathrm{~s}^{-1} ;(b) \omega=1.78 \cdot 10^{15} \mathrm{~s}^{-1}$.

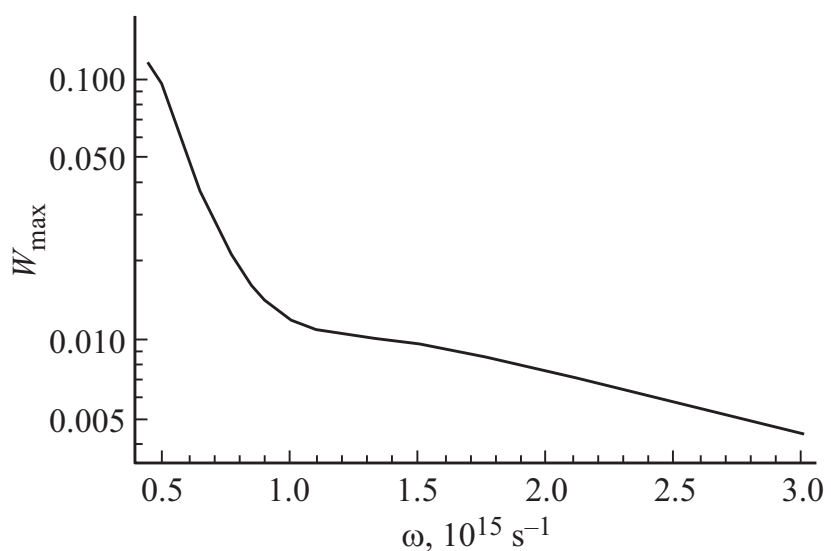

Рис. 5. Зависимость $W_{\max }$ от частоты света $\omega$ при $j_{\omega}=100 \mathrm{GW} / \mathrm{cm}^{2}$.

зоне интенсивностей $j_{\omega}$ этот вклад оказывается весьма существенным. Следует, однако, отметить, что провалы на кривых $W_{\max }\left(j_{\omega}\right)$ и $W_{\max }(\omega)$ (рис. 6) появляются в результате экстраполяции результатов расчетов на область частот $\omega$ и интенсивностей $j_{\omega}$, где условия (16) перестают выполняться.

\section{5. Обсуждение результатов}

При высоких интенсивностях света, как видно из приведенных в предыдущем разделе зависимостей, однофотонное внутризонное поглощение (процессы с изменением энергии электрона на $(\hbar \omega)$ претерпевает радикальные изменения по сравнению со случаем умеренных интенсивностей. Фактически поглощение идет только до времени достижения первого максимума $\tau_{1}$ (рис. 1,3 ). Затем происходит периодическое перекачивание энергии между электронной подсистемой и электромагнитным полем. Так, например, при $j_{\omega}=1.5 \mathrm{TW} / \mathrm{cm}^{2}$ и $\omega=1.78 \times 10^{15} \mathrm{~s}^{-1}$ получим $\tau_{1} \approx 150 \mathrm{fs}$, причем зависимость $W_{\text {ехс }}$ от $t$ заметно отклоняется от линейной уже при временах действия импульса, больших $20 \mathrm{fs}$. Зависимость $W_{\text {exc }}$ от $j_{\omega}$ при временах действия лазерного импульса от единиц до десятков fs также сильно отличается от линейной (см., например, рис. 7). 

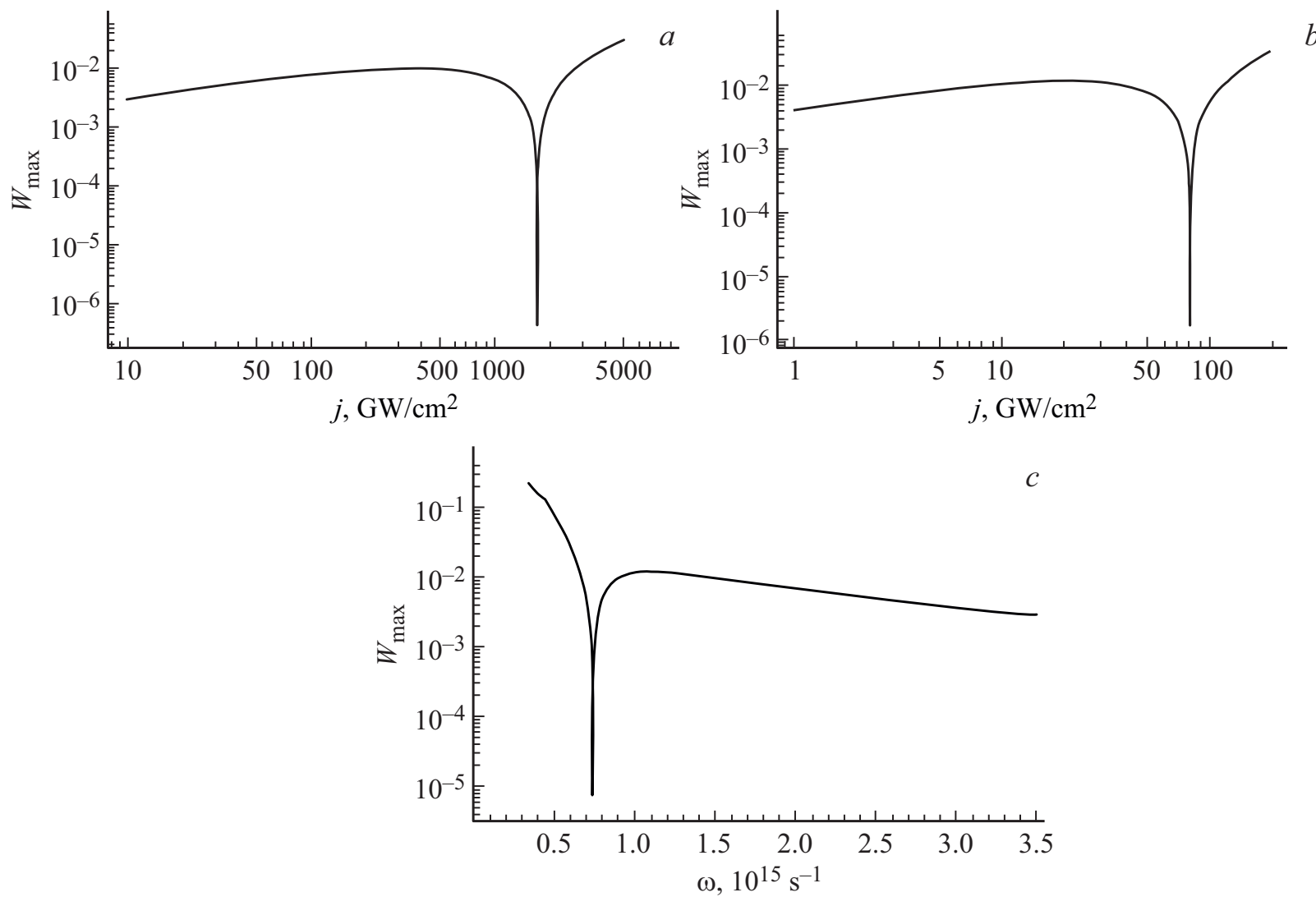

Рис. 6. Зависимости максимальной вероятности $W_{\max }^{\operatorname{mono}}$ от интенсивности $j_{\omega}:(a)$ при $\omega=0.7 \cdot 10^{15} \mathrm{~s}^{-1},(b)$ при $\omega=1.78 \cdot 10^{15} \mathrm{~s}^{-1}$; (c) от частоты $\omega$ при $j_{\omega}=100 \mathrm{GW} / \mathrm{cm}^{2}$.

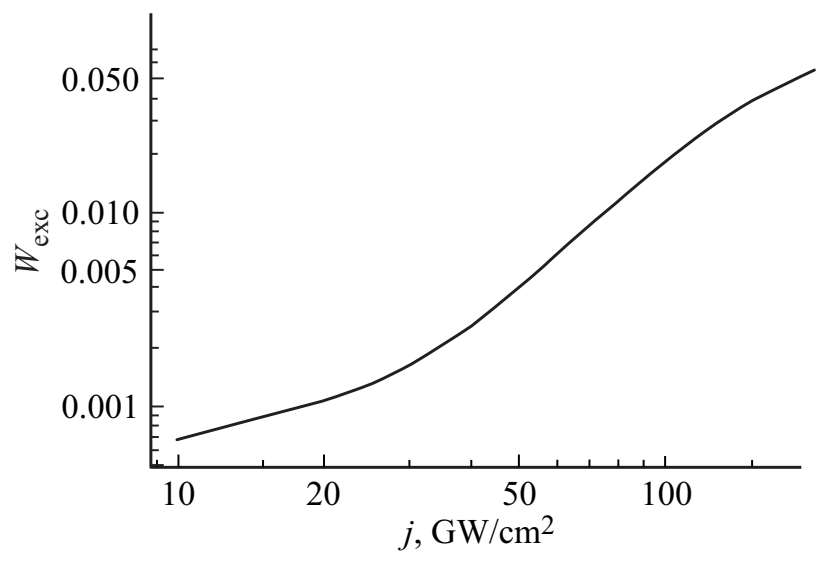

Рис. 7. Зависимость $W_{\text {ехс }}$ от интенсивности $j_{\omega}$ для времени действия лазерного импульса $t=20 \mathrm{fs}, \omega=0.7 \cdot 10^{15} \mathrm{~s}^{-1}$. Значения остальных параметров указаны в начале разд. 4 .

Представленная в данной работе теория описывает в первую очередь случай очень высоких интенсивностей лазерного излучения. В то же время в случае умеренных интенсивностей света $j_{\omega}$ расчет по формуле (28) приводит к линейной зависимости $W_{\text {exс }}$ как от времени (рис. $8, a)$, так и от интенсивности $j_{\omega}$ (рис. $\left.8, b\right)$. В этом случае можно ввести коэффициент поглощения

$$
\alpha=n_{0} W_{\mathrm{exc}}(t) \hbar \omega /\left(j_{\omega} t\right),
$$

где $n_{0}$ - концентрация электронов в зоне проводимости. При частоте света $\omega=1.78 \times 10^{15} \mathrm{~s}^{-1}, n_{0}=10^{17} \mathrm{~cm}^{-3}$ и для указанных выше значений параметров кристалла $\alpha \approx 0.043 \mathrm{~cm}^{-1}$, что является весьма разумным значением.

Следует иметь в виду, что для получения сравнительно простого выражения (28) в работе использовалась весьма упрощенная модель процесса нелинейного поглощения лазерного излучения свободными электронами в зоне проводимости. Отметим ряд важных факторов.

- Не учитывалась анизотропия электронного зонного спектра.

- Зона проводимости считалась достаточно узкой, так что электроны не могли переходить в зонные состояния с энергией $E_{k} \sim n \hbar \omega(n>1)$.

- В работе был рассмотрен только механизм поглощения с участием LO-фононов, взаимодействие электронов с которыми описывалось гамильтонианом Фрёлиха. В то же время вклад в поглощение могут дать механизмы, где электрон-фононное взаимодействие описывается оптическим деформационным потенциалом, либо механизмы с участием акустических фононов или с рассеянием на заряженных примесях. 

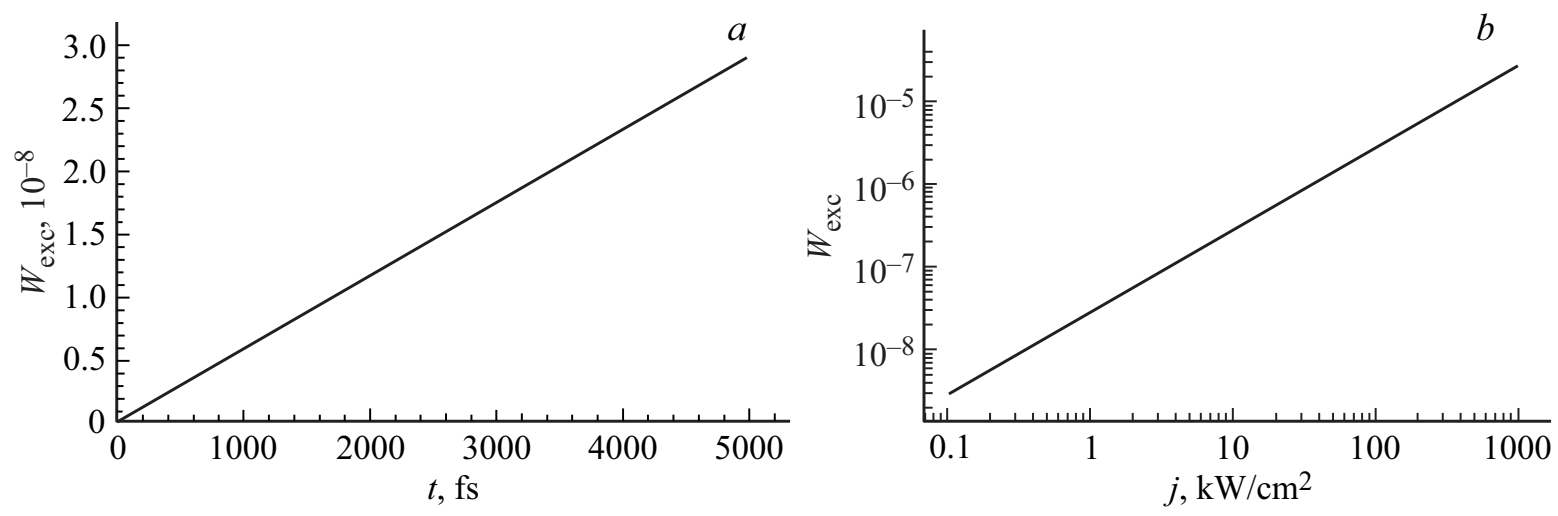

Рис. 8. Линейная зависимость $W_{\text {ехс}}$ : $(a)$ от времени при умеренной интенсивности света $j_{\omega}=1 \mathrm{~kW} / \mathrm{cm}^{2} ;(b)$ от интенсивности, $t=5$ ps. В обоих случаях $\omega=0.7 \cdot 10^{15} \mathrm{~s}^{-1}$.

- Фактически рассматривались лазерные импульсы прямоугольной формы, хотя при длительностях импульсов порядка единиц или десятков фемтосекунд учет формы лазерного импульса заметно (хотя и некритическим образом) влияет на результат (см., например, [30]).

- Не учитывалось реальное распределение интенсивностей по поперечному сечению светового пучка.

Тем не менее при условии $P_{s} \tau \gg 1$, которое достигается при интенсивностях $j_{\omega} \sim 10-100 \mathrm{GW} / \mathrm{cm}^{2}$, однофотонное поглощение будет ограничено временами достижения первого пика на зависимостях $W_{\text {exc }}(t)$. Это обстоятельство существенно снижает эффективность ступенчатого внутризонного возбуждения свободных электронов и проявляется в увеличении роли многофотонных процессов (МФП). В рамках развитого подхода могут быть рассмотрены и непрямые внутризонные МФП. В этом случае будут иметь место $(l+1)$-квантовые осцилляции Раби для $l$-фотонных переходов.

\section{6. Заключение}

В работе в рамках упрощенной модели рассмотрено поглощение лазерного излучения на свободных электронах в зоне проводимости неметаллического кристалла. Получено простое выражение для вероятности перехода $W_{\text {exс }}$ из состояний электрона вблизи дна зоны проводимости в состояния с энергией, большей на величину светового кванта. Показано, что имеют место специфические электрон-фононные осцилляции Раби, а поглощение света идет только до времени достижения первого максимума $\tau_{1}$ на зависимости $W_{\text {exс }}$ от времени $t$ с начала действия лазерного импульса. Получены нелинейные зависимости максимальных значений $W_{\text {exс }}$ от интенсивности света $j_{\omega}$ при фиксированной частоте света $\omega$ и от $W_{\text {exс }}$ при фиксированном значении $j_{\omega}$. Получены также зависимости $\tau_{1}$ и частоты фотон-фононных осцилляций Раби $\Omega_{\mathrm{R}}$ от $j_{\omega}$ при нескольких значениях $\omega$. Показано, что процессы высоких порядков по полю световой волны в рассматриваемом диапазоне предпробойных интенсивностей света существенным образом влияют на $W_{\mathrm{exc}}, \tau_{1}$ и $\Omega_{\mathrm{R}}$. В предельном случае слабых полей полученные формулы описывают линейное поглощение света и дают разумное значение коэффициента поглощения.

\section{Финсансирование работы}

Работа выполнена при государственной поддержке ведущих университетов Российской Федерации (субсидия 08-08).

\section{Конфликт интересов}

Авторы заявляют, что у них нет конфликта интересов.

\section{Список литературы}

[1] Маненков А.А., Прохоров А.М. // УФН. 1986. Т. 148. № 1. C. 179; Manenkov A.A., Prokhorov A.M. // Sov. Phys. Usp. 1986. V. 29. N 1. P. 104. doi 10.1070/PU1986v029n01ABEH003117

[2] Jones S.C., Braunlich P., Casper R.T., Shen X.-A., Kelly P. // Opt. Engin. 1989. V. 28. N 10. 281039. doi 10.1117/12.7977089

[3] Lenzner M., Krüger J., Sartania S., Cheng Z., Spielmann C., Mourou G., Kautek W., Krausz F. // Phys. Rev. Lett. 1998. V. 80. N 18. P. 4076. doi 10.1103/PhysRevLett.80.4076

[4] Stuart B.C., Feit M.D., Herman S., Rubenchik A.M., Shore B.W., Perry M.D. // Phys. Rev. B. 1996. V. 53. N 4. P. 1749. doi 10.1103/PhysRevB.53.1749

[5] Simanovskii D.M., Schwettman H.A., Lee H., Welch A.J. // Phys. Rev. Lett. 2003. V. 91. N 10. 107601. doi 10.1103/PhysRevLett.91.107601

[6] Bulgakova N.M., Stoian R., Rosenfeld A., Hertel I.V., Campbell E.E.B. // Phys. Rev. B 2004. V. 69. N 5. 054102. doi 10.1103/PhysRevB.69.054102

[7] Deng Y.P., Xie X.H., Xiong H., Leng Y.X., Cheng C.F., Lu H.H., Li R.X., Xu Z. // Opt. Express. 2005. V. 13. N 8. P. 3096. doi 10.1364/OPEX.13.003096

[8] Rethfeld B. // Phys. Rev. B. 2006. V. 73. N 3. 035101. doi 10.1103/PhysRevB.73.035101 
[9] Bachau H., Belsky A.N., Bogatyrev I.B., Gaudin J., Geoffroy G., Guizard S., Martin P., Popov Yu.V., Vasil'ev A.N., Yatsenko B.N. // Appl. Phys. A. 2010. V. 98. P. 679. doi 10.1007/s00339-009-5465-y

[10] Chen H.Y., Wan Z.Z., Han Y.L. // Appl. Mechanics and Materials. 2011. V. 142. P. 134. doi 10.4028/www.scientific.net/amm.142.134

[11] Mirza I., Bulgakova N.M., Tomáštík J., Michálek V., Haderka O., Fekete L., Mocek T. // Sci. Rep. 2016. V. 6. P. 39133. doi 10.1038/srep39133

[12] Lin Zh., Ji L., Hong M. // Photonics Research. 2020. V. 8. N 3. P. 271. doi 10.1364/PRJ.379254

[13] Перлин Е.Ю., Федоров А.В., Камевник М.Б. // ЖЭТФ. 1983. T. 85. № 4(10). C. 1357; Perlin E.Yu., Fedorov A.V., Kashevnik M.B. // Sov. Phys. JETP. 1983. V. 58. N 4. P. 787.

[14] Перлин Е.Ю., Иванов А.В., Левицкий Р.С. // ЖЭТФ. 2005. T. 128. № 2(8). C. 411; Perlin E.Yu., Ivanov A.V., Levitskii R.S. // JETP. 2005. V. 101. N 2. P. 357. doi $10.1134 / 1.2047802$

[15] Fan H.Y., Spitzer W., Collins R.J. // Phys. Rev. 1956. V. 101. N 2. P. 567. doi $10.1016 / 0038-1098(86) 90279-6$ (Eq. (5) is incorrect)

[16] Seeger K. Semiconductor Physics. Wien, NY.: Springer, 1973 (Chapter 11); Зеегер К. Физика полупроводников. М.: Мир, 1977. $616 \mathrm{c}$.

[17] Ansel'm A.I. Introduction to Semiconductor Theory. Upper Saddle River, NJ: Prentice-Hall, 1982; Ансельм А.И. Введение в теорию полупроводников. М.: Наука, 1978. 616 с.

[18] Ridley B.K. Quantum processes in semiconductors. Clarendon Press. Oxford, 1982; Ридли Б. Квантовые процессы в полупроводниках. М.: Мир, 1986.

[19] Осипова М.О., Перлин Е.Ю. // Опт. журн. 2016. Т. 83. № 11. C. 3; Osipova M.O., Perlin E.Yu. // J. Opt. Technol. 2016. V. 83. N 11. P. 648. doi 10.1364/JOT.83.000648

[20] Жукова М.О., Перлин Е.Ю. // ОПт. журн. 2017. Т. 84. № 10. C. 3-6; Zhukova M.O., Perlin E.Yu. // J. Opt. Technol. 2017. V. 84. N 10. P. 651. doi 10.1364/JOT.84.000651

[21] Леонов М.Ю., Орлова А.О., Баранов А.В., Рухленко И.Д., Гунько Ю.К., Федоров А.В. // Опт. журн. 2013. Т. 80. В. 11. C. 7; Leonov M.Yu., Orlova A.O., Baranov A.V., Fedorov A.V., Rukhlenko I.D., Gun'ko Yu.K. // J. Opt. Technol. 2013. V. 80. N 11. P. 648. doi 10.1364/JOT.80.000648

[22] Pellemans H.P.M., Planken P.C.M. // Phys. Rev. B. 1998. V. 57. N 8. R4222. doi 10.1103/PhysRevB.57.R4222

[23] Peelaers H., Van de Walle C.G. // Phys. Rev. B. 2019. V. 100. 081202(R). doi 10.1103/PhysRevB.100.081202

[24] Малевич В.Л., Эпштейн Э.М. // Квант. электрон. 1974. T. 1. № 6. C. 1468; Malevich V.L., Epshtein E.M. // Sov. J. Quant. Electron. 1974. V. 4. N 6. P. 1816. doi 10.1070/QE1974v004n06ABEH009345

[25] Fomin V.M., Pokatilov E.P. // Phys. Stat. Sol. B. 1976. V. 78. N 2. P. 831. doi 10.1002/pssb.2220780244

[26] Fomin V.M., Pokatilov E.P. // Phys. Stat. Sol. B. 1983. V. 119. N 2. P. 483. doi $10.1002 /$ pssb. 2221190206

[27] Apostolova T., Huang D.H., Alsing P.M., McIver J., Cardimona D.A. // Phys. Rev. B. 2002. V. 66. 075208. doi 10.1103/PhysRevB.66.0752

[28] Huang D., Apostolova T., Alsing P.M., Cardimona D.A. // Phys. Rev. B. 2004. V. 69. 075214. doi 10.1103/PhysRevB.69.075214
[29] Huang D., Alsing P.M., Apostolova T., Cardimona D.A. // Phys. Rev. B. 2005. V. 71.045204. doi 10.1103/PhysRevB.71.045204

[30] Перлин Е.Ю., Бондарев М.А., Жукова М.О. // Опт. и спектр. 2017. Т. 123. № 4. С. 566. $\begin{array}{lllll}\text { doi } & 10.7868 / \mathrm{S} 0030403417100178 & \text { (I), } & \text { C. } & 571 .\end{array}$ doi 10.7868/S003040341710018X (II); Perlin E.Yu., Bondarev M.A., Zhukova M.O. // Opt. Spectrosc. 2017. V. 123. N 4. P. 578. doi 10.1134/S0030400X17100174, P. 583 (II). doi 10.1134/S0030400X17100186

[31] Autler S.H., Townes C.H. // Phys. Rev. 1955. V. 100. N 2. P. 703. doi 10.1103/PhysRev.100.703

[32] Yacoby Y. // Phys. Rev. B. 1970. V. 1. N 4. P. 1666. doi 10.1103/PhysRevB.1.1666

[33] Галищкий В.М., Гореславский С.П., Елесин В.Ф. // ЖЭТФ. 1970. T. 57. № 1. C. 207; Galitskii V.M., Goreslavskii S.P., Elesin V.F. // JETP. 1970. V. 30. N 1. P. 117.

[34] Перлин Е.Ю., Коварский В.А. // ФТТ. 1970 Т. 17. № 11. C. 3105; Perlin E.Yu., Kovarskii V.A. // Sov. Phys. Solid State. 1970. V. 12. P. 2512.

[35] Tzoar N., Gersten J.I. // Phys. Rev. B. 1975. V. 12. N 4. P. 1132. doi 10.1103/PhysRevB.12.1132

[36] Перлин Е.Ю. // ФТТ. 1973. Т. 15. № 1. С. 66; Perlin E.Yu. // Fiz. Tverd. Tela (Leningrad). 1973. V. 15. N 1. P. 66.

[37] Балкарей Ю.И., Эпштейн Э.М. // ФТТ. 1973. Т. 15. № 3. C. 925.

[38] Перлин Е.Ю. // Опт. и спектр. 1976. Т. 41. № 2. С. 263; Perlin E.Yu. // Opt. Spectrosc. 1976. V. 41. N 8. P. 153.

[39] Fröhlich D., Nothe A., Reimann K. // Phys. Rev. Lett. 1985. V. 55. N 12. P. 1335. doi 10.1103/PhysRevLett.55.1335

[40] Von Lehmen A., Chemla D.S., Heritage J.P., Zucker J.E. // Opt. Lett. 1986. V. 11. N 10. P. 609. doi 10.1364/OL.11.000609

[41] Mysyrowicz A., Hulin D., Antonetti A., Migus A., Masselink W.T., Morko H. // Phys. Rev. Lett. 1986. V. 56. N 25. P. 2748. doi 10.1103/PhysRevLett.56.2748

[42] Schäfer W., Schuldt K.H., Binder R. // Phys. Stat. Sol. B. 1988. V. 150. N 2. P. 407. doi 10.1002/pssb.2221500209

[43] Knox W.H., Chemla D.S., Miller D.A.B., Stark J.B., SchmittRink S. // Phys. Rev. Lett. 1989. V. 62. N 10. P. 1189. doi 10.1103/PhysRevLett.62.1189

[44] Chemla D.S., Knox W.H., Miller D.A.B., Schmitt-Rink S., Stark J.B., Zimmermann R. // J. Luminesc. 1989. V. 44. N 4. P. 233. doi 10.1016/0022-2313(89)90060-4

[45] Перлин Е.Ю., Федоров А.В. // Опт. и спектр. 1995. Т. 78. № 3. C. 445; Perlin E.Yu., Fedorov A.V. // Opt. Spectrosc. 1995. V. 78. N 3. P. 400.

[46] Перлин Е.Ю., Федоров А.В. // ФТТ. 1995. Т. 37. № 5. C. 1463; Perlin E.Yu., Fedorov A.V. // Phys. Solid State. 1995. V. 37. N 5. P. 792.

[47] Перлин Е.Ю. // ЖЭТФ. 1994. Т. 78. № 1. С. 186; Perlin E.Yu. // JETP. 1994. V. 78. N 1. P. 98.

[48] Перлин Е.Ю. // Опт. и спектр. 1997. Т. 83. № 2. С. 262; Perlin E.Yu. // Opt. Spectrosc. 1997. V. 83. N 2. P. 243. doi 10.1134/1.1953976

[49] Unold T., Mueller K., Lienau C., Elsaesser T., Wieck A.D. // Phys. Rev. Lett. 2004. V. 92. N 15. 157401. doi 10.1103/PhysRevLett.92.157401

[50] Перлин Е.Ю., Стаселько Д.И. // Опт. и спектр. 2005. Т. 98. № 6. C. 944; Perlin E.Yu., Stasel'ko D.I. // Opt. Spectrosc. 2005. V. 98. N 6. P. 844. doi 10.1134/1.1953976 
[51] Mizumoto Y., Kayanuma Y., Srivastava A., Kono J., Chin A.H. // Phys. Rev. B. 2006. V. 74. N 4.045216. doi 10.1103/PhysRevB.74.045216

[52] Иванов А.В., Перлин Е.Ю. // ОПт. и спектр. 2009. T. 106. № 5. C. 56 (I), 764 (II); Ivanov A.V., Perlin E.Yu. // Opt. Spectrosc. 2009. V. 106. N 5. P. 677. doi 10.1134/S0030400X09050099 (I), P. 685. doi 10.1134/S0030400X09050105 (II).

[53] Бондарев М.А., Перлин Е.Ю. // ОПт. и спектр. 2017. T. 122. № 4. C. 580. (I), 586. (II); Bondarev M.A., Perlin E.Yu. // Opt. Spectrosc. 2017. V. 122. N 4. P. 561. doi 10.1134/S0030400X17040063 (I), P. 567. doi 10.1134/S0030400X17040075 (II)

[54] Sim S., Lee D., Noh M., Cha S., Soh C.H., Sung J.H., Jo M.-H., Choi H. // Nat. Commun. 2016. V. 7. 13569. doi $10.1038 /$ ncomms 13569

[55] Ivanov A. // JOSA B. 2018. V. 35. N 1. P. 20. doi 10.1364/JOSAB.35.000020

[56] Yong C.-K., Horng J., Shen Y., Cai H., Wang A., Yang C.S., Lin C.-K., Zhao S., Watanabe K., Taniguchi T., Tongay S., Wang F. // Nature Physics. 2018. V. 14. P. 1092. doi 10.1038/s41567-018-0216-7

[57] Lamountain T., Bergeron H., Balla I., Stanev T.K., Hersam M.C., Stern N.P. // Phys. Rev. B. 2018. V. 97. 045307. doi 10.1103/PhysRevB.97.045307

[58] Cunningham P.D., Hanbicki A.T., Reinecke T.L., McCreary K.M., Jonker B.T. //. Nat. Commun. 2019. V. 10. 5539. doi 10.1038/s41467-019-13501-x

[59] Fano U. // Phys. Rev. 1961. V. 124. P. 1866. doi 10.1103/PhysRev.124.1866

[60] Акулин В.М., Карлов Н.В. Интенсивные резонансные взаимодействия в квантовой электронике. М.: Наука, 1987. 2 c.; Akulin V.M., Karlov N.V. Intense Resonant Interactions in Quantum Electronics. Berlin: Springer, 1992. Lecture 9.

[61] Linskens A.F., Holleman I., Dam N., Reuss J. // Phys. Rev. A. 1996. V. 54. N 6. P. 4854. doi 10.1103/PhysRevA.54.4854

[62] Kittel C. Quantum Theory of Solids. NY.: Wiley, 1987. 528 p.; Киттель Ч. Квантовая теория твердых тел. М.: Наука, 1967. $492 \mathrm{c}$

[63] Handbook of Mathematical Functions with Formulas, Graphs, and Mathematical Tables / Ed. by Abramovitz M., Stegun I.A. National Bureau of Standards, Appl. Math. Ser. - 55. 1964; Справочник по специальным функциям с формулами, графиками и математическими таблицами / Под ред. Абрамовитца М., Стиган И. М.: Наука, 1979. 